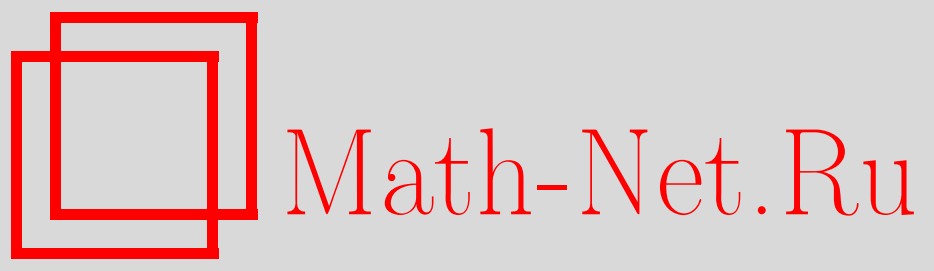

С. Н. Лакаев, С. С. Улашов, Существование и аналитичность связанных состояний двухчастичного оператора Шредингера на решетке, ТMФ, 2012, том 170, номep 3, 393-408

DOI: https://doi.org/10.4213/tmf6774

Использование Общероссийского математического портала Math-Net.Ru подразумевает, что вы прочитали и согласны с пользовательским соглашением http: //www . mathnet.ru/rus/agreement

Параметры загрузки:

IP : 34.227 .88 .159

26 апреля 2023 г., $17: 45: 33$

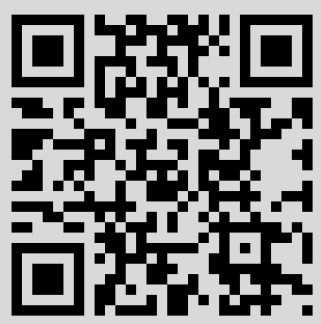




\title{
СУЩЕСТВОВАНИЕ И АНАЛИТИЧНОСТЬ СВЯЗАННЫХ СОСТОЯНИЙ ДВУХЧАСТИЧНОГО ОПЕРАТОРА ШРЕДИНГЕРА НА РЕШЕТКЕ
}

\begin{abstract}
Рассмотрен двухчастичный дискретный оператор Шредингера $H_{\mu}(K)$, соответствующий системе двух произвольных частиц на $d$-мерной решетке $\mathbb{Z}^{d}$, $d \geqslant 3$, которые взаимодействуют с помощью парного контактного потенциала отталкивания с константой связи $\mu>0$ ( $K \in \mathbb{T}^{d}-$ квазиимпульс двух частиц). Установлено, что верхний (правый) край существенного спектра может быть либо виртуальным уровнем (при $d=3,4$ ), либо собственным значением (при $d \geqslant 5)$ оператора $H_{\mu}(K)$. Показано, что существует единственное собственное значение, лежащее правее существенного спектра, в зависимости от значений константы связи $\mu$ и двухчастичного квазиимпульса $K$. Доказаны аналитичность соответствующего собственного состояния, а также аналитичности собственного значения и собственного состояния как функций квазиимпульса $K \in \mathbb{T}^{d}$ в области их существования.
\end{abstract}

Ключевые слова: дискретный оператор Шредингера, система двух частиц, гамильтониан, контактный отталкивающий потенциал, виртуальный уровень, собственное значение, решетка.

\section{1. ВВЕДЕНИЕ}

Кинематика квантовых квазичастиц на решетке довольно экзотична даже в случае двух частиц. В частности, благодаря тому что дискретный аналог лапласиана не является трансляционно-инвариантным, гамильтониан системы не разделяется на две части: одну, относящуюся к движению центра масс, и другую, относящуюся ко внутренним степеням свободы. В системах на решетке нет такой простой характеристики инерции, как масса, но возможна соответствующая локальная характеристика - эффективная масса. Кроме того, такой естественный локальный заменитель массы, как эффективный тензор масс связанного состояния, зависит от квазиимпульса системы и является только полуаддитивным. Это так называемое явление избытка масс для систем на решетке: эффективная масса $N$-частичного связанного состояния больше, чем сумма эффективных масс квазичастиц, составляющих систему [1], [2].

* Самаркандский государственный университет, Самарканд, Узбекистан. E-mail: slakaev@mail.ru 
Двухчастичная задача на решетке, в отличие от непрерывного случая, где отделяется движение центра масс, сводится к изучению одночастичной задачи с помощью преобразования Гельфанда: гильбертово пространство $l_{2}\left(\left(\mathbb{Z}^{d}\right)^{2}\right)$ разлагается в прямой (непрерывный) интеграл фон Неймана, ассоциированный с представлением абелевой (дискретной) группы $\mathbb{Z}^{d}$, которая образована с помощью перестановочных операторов на решетке. Тогда двухчастичный гамильтониан $H_{\mu}$ также разлагается в прямой (непрерывный) интеграл фон Неймана [3]:

$$
H_{\mu}=\int_{\mathbb{T}^{d}} \oplus H_{\mu}(K) d K
$$

где $K$ пробегает первую зону Бриллюэна $\mathbb{T}^{d}=\mathbb{R}^{\nu} /(2 \pi \mathbb{Z})^{d}$. В отличие от непрерывного случая, соответствующие слойные операторы $H_{\mu}(K)$ в разложении в прямой интеграл параметрически зависят от квазиимпульса $K \in \mathbb{T}^{d}$. В результате вследствие того, что задача не является сферически-симметричной, спектры операторов $H_{\mu}(K)$ оказываются довольно чувствительными к изменению квазиимпульса $K$.

В спектральном анализе непрерывного оператора Шредингера $H_{\mu}=-\Delta+\mu V$ в $\mathbb{R}^{3}$ наблюдается появление собственного значения из непрерывного спектра для критических потенциалов (при пороговых значениях константы связи $\mu>0$ ) [4]-[8]. Это явление связано с решением уравнения Шредингера, которое убывает на бесконечности, но не является квадратично-интегрируемым. Такие решения обычно называются резонансными состояниями (функциями) с нулевой энергией, и в этом случае оператор Шредингера $H_{\mu_{0}}$ называется критическим или имеющим резонанс с нулевой энергией (виртуальный уровень). Появление собственного значения у неотрицательного критического оператора Шредингера $H_{\mu}$ с пороговым значением константы связи $\mu=\mu_{0}>0$ при малых возмущениях особенно замечательно: если в некоторой трехчастичной системе никакая подсистема не имеет связанного состояния и не менее чем две его двухчастичные подсистемы имеют резонанс с нулевой энергией, то трехчастичная система обладает бесконечным числом связанных состояний с отрицательными энергиями, стремящимся к нулю, т. е. имеет место эффект Ефимова [5], [7]-[12].

Пусть $\mathbf{H}_{\mu}(K)$ - трехчастичный решетчатый оператор Шредингера $\left(K \in \mathbb{T}^{3}-\right.$ трехчастичный квазиимпульс), ассоциированный с гамильтонианом системы трех одинаковых частиц, которые движутся на $\mathbb{Z}^{3}$ и взаимодействуют с помощью парных контактных потенциалов притяжения с константой связи $\mu>0$. Следующее явление также тесно связано с пороговым значением константы связи $\mu=\mu_{0}>0$ двухчастичных операторов: при $\mu=\mu_{0}$ соответствующий трехчастичный оператор Шредингера $\mathbf{H}_{\mu_{0}}(0)$ имеет бесконечное число собственных значений, однако оператор $\mathbf{H}_{\mu_{0}}(K)$ при $K \neq 0$ имеет только конечное число собственных значений [5], [12].

В работе [3] для широкого класса двухчастичных дискретных операторов Шредингера $H_{\mu}(K), K \in \mathbb{T}^{d}, d \geqslant 3$, ассоциированных с гамильтонианом системы двух произвольных частиц, которые взаимодействуют с помощью парных короткодействующих потенциалов, показано следующее. Если при некотором значении $\mu=$ $\mu_{0}>0$ неотрицательный оператор $H_{\mu_{0}}(0)$ имеет виртуальный уровень или собственное значение на дне (левом краю) существенного спектра, то при всех ненулевых значениях квазиимпульса $K \in \mathbb{T}^{d}$ оператор $H_{\mu_{0}}(K)$ имеет собственное значение, лежащее ниже дна существенного спектра.

В физике устойчивые сложные объекты обычно образуются с помощью сил притяжения, которые позволяют составным частям уменьшить энергию, связывая их 
вместе. Отталкивающие силы отделяют частицы в свободном пространстве. Однако в работе [13] описано экспериментальное наблюдение, состоящее в том, что в упорядоченных средах с периодическим потенциалом, а также в отсутствие рассеяния устойчивые сложные объекты могут существовать даже в случае отталкивающих взаимодействий.

Следует отметить, что в работе [14] изучена проблема существования связанных состояний оператора $H_{\mu}(K)$ при некоторых значениях квазиимпульса $K \in \mathbb{T}^{3}$. В частности, установлено, что оператор $H_{\mu}(\vec{\pi})$ не имеет собственных значений при малых $\mu>0$.

В настоящей работе изучены спектральные свойства оператора Шредингера $H_{\mu}(K), K \in \mathbb{T}^{d}$, ассоциированного с гамильтонианом системы двух произвольных частиц, которые взаимодействуют с помощью парных контактных потенциалов с константой связи $\mu>0$, в зависимости как от константы парного взаимодействия отталкивания $\mu>0$, так и от квазиимпульса системы $K \in \mathbb{T}^{d}$. Показано, что:

1) оператор Шредингера $H_{\mu}(K)$ имеет пороговый виртуальный уровень (пороговое собственное значение) не только при нулевом значении квазиимпульса $K \in \mathbb{T}^{d}$, но и при всех других значениях $K \in \mathbb{T}^{d}$, в зависимости от значения константы контактного потенциала отталкивания $\mu$;

2 ) для любого $K \in \mathbb{T}^{d}$ существует $\mu(K)>0$ такое, что при $0<\mu<\mu(K)$ оператор $H_{\mu}(K)$ не имеет собственного значения, лежащего правее существенного спектра, а при $\mu>\mu(K)>0$ он имеет единственное собственное значение.

При фиксированном значении константы связи $\mu>0$, рассматривая пороговое значение константы связи как функцию $\mu(K)>0$, определенную на торе $\mathbb{T}^{d}$, мы приходим к следующему разбиению тора: $\mathbb{T}^{d}=M_{<}(\mu) \cup M_{=}(\mu) \cup M_{>}(\mu)$, где $M_{<}(\mu)$ и $M_{>}(\mu)$ - открытые связные множества, а $M_{=}(\mu)$ - замкнутое многообразие коразмерности 1. При этом для любого $K \in M_{=}(\mu)$ оператор $H_{\mu}(K)$ имеет пороговый виртуальный уровень (при $d=3,4$ ) или пороговое собственное значение (при $d \geqslant 5$ ); при всех $K \in M_{<}(\mu)$ оператор $H_{\mu}(K)$ имеет единственное собственное значение, лежащее правее существенного спектра $\sigma_{\mathrm{ess}}\left(H_{\mu}(K)\right)$, а при всех $K \in M_{>}(\mu)$ не имеет собственного значения. Соответствующее собственное состояние $\psi_{\mu, K}(\cdot), K \in M_{<}(\mu)$, является аналитической функцией на торе $\mathbb{T}^{d}$. Отображение $\psi_{\mu}: M_{<}(\mu) \mapsto L^{2}\left(\mathbb{T}^{d}\right)$ такое, что $K \mapsto \psi_{\mu, K}(\cdot)$, является векторнозначным аналитическим отображением на $M_{<}(\mu)$. Установлено, что собственное значение $E_{\mu}(\cdot)$ - аналитическая функция в $M_{<}(\mu)$, а также найдены верхняя и нижняя оценки его положения.

\section{2. ОПЕРАТОРЫ ЭНЕРГИИ ДВУХ ЧАСТИЦ НА РЕШЕТКЕ. КООРДИНАТНОЕ И ИМПУЛЬСНОЕ ПРЕДСТАВЛЕНИЯ}

2.1. Координатное представление. Пусть $\mathbb{Z}^{d}-d$-мерная целочисленная решетка и $\left(\mathbb{Z}^{d}\right)^{2}$ - декартово произведение решеток. Обозначим через $l_{2}\left(\left(\mathbb{Z}^{d}\right)^{2}\right)$ гильбертово пространство квадратично-суммируемых функций, определенных на $\left(\mathbb{Z}^{d}\right)^{2}$. Свободный гамильтониан $\widehat{H}_{0}$ рассматриваемой системы двух произвольных частиц (бозона и фермиона) в координатном представлении на $d$-мерной решетке $\mathbb{Z}^{d}$ определяется как ограниченный самосопряженный оператор, действующий в гильбертовом пространстве $l_{2}\left(\left(\mathbb{Z}^{d}\right)^{2}\right)$ :

$$
\widehat{H}_{0}=\frac{1}{2 m_{1}} \Delta_{x_{1}}+\frac{1}{2 m_{2}} \Delta_{x_{2}}
$$


где $\Delta_{x_{1}}=\Delta \otimes I$ и $\Delta_{x_{2}}=I \otimes \Delta$, а $m_{\alpha}>0$ обозначает массу частиц, $\alpha=1,2$. Лапласиан $\Delta$ на решетке есть разностный оператор, описывающий перенос частицы с узла на соседний узел:

$$
(\Delta \widehat{\psi})(x)=\sum_{|s|=1}[\widehat{\psi}(x)-\widehat{\psi}(x+s)], \quad \widehat{\psi} \in l_{2}\left(\mathbb{Z}^{d}\right) .
$$

Здесь $s=\left(s^{(1)}, \ldots, s^{(d)}\right) \in \mathbb{Z}^{d},|s|=\left|s^{(1)}\right|+\cdots+\left|s^{(d)}\right|$. Заметим, что $\widehat{H}_{0}$ действует в пространстве $l_{2}\left(\left(\mathbb{Z}^{d}\right)^{2}\right)$ по формуле

$$
\left(\widehat{H}_{0} \widehat{\psi}\right)\left(x_{1}, x_{2}\right)=\sum_{s \in \mathbb{Z}^{d}} \hat{\varepsilon}(s)\left[\widehat{\psi}\left(x_{1}+s, x_{2}\right)+\gamma \widehat{\psi}\left(x_{1}, x_{2}+s\right)\right], \quad \widehat{\psi} \in l_{2}\left(\left(\mathbb{Z}^{d}\right)^{2}\right),
$$

где $\gamma=m_{1} / m_{2}>0$ и функция $\hat{\varepsilon}(\cdot)$ в $\mathbb{Z}^{d}$ определяется как

$$
\hat{\varepsilon}(s)= \begin{cases}d, & s=0 \\ -1 / 2, & |s|=1 \\ 0, & |s|>1\end{cases}
$$

Полный двухчастичный гамильтониан $\widehat{H}_{\mu}$ описывает взаимодействие между двумя частицами и определяется как ограниченный самосопряженный оператор $\widehat{H}_{\mu}=$ $\widehat{H}_{0}+\mu \widehat{V}$, действующий в гильбертовом пространстве $l_{2}\left(\left(\mathbb{Z}^{d}\right)^{2}\right)[15]$. Здесь $\widehat{V}-$ оператор умножения на функцию,

$$
(\widehat{V} \widehat{\psi})\left(x_{1}, x_{2}\right)=\delta_{x_{1} x_{2}} \widehat{\psi}\left(x_{1}, x_{2}\right), \quad \widehat{\psi} \in \ell^{2}\left(\left(\mathbb{Z}^{d}\right)^{2}\right),
$$

и $\delta_{x_{1} x_{2}}-$ символ Кронекера.

2.2. Импульсное представление. Пусть $\mathbb{T}^{d}-d$-мерный тор, т. е. куб $(-\pi, \pi]^{d}$ с соответствующим отождествлением противоположных граней. Он рассматривается как абелева группа, в которой операции сложения и умножения на вещественное число введены как операции сложения и умножения на вещественное число в $\mathbb{R}^{d}$ по модулю $(2 \pi \mathbb{Z})^{d}$. Пусть $L^{2}\left(\left(\mathbb{T}^{d}\right)^{2}\right)$ - гильбертово пространство квадратично-интегрируемых функций, определенных на $\left(\mathbb{T}^{d}\right)^{2}$ и $\widehat{\mathcal{F}}: L^{2}\left(\left(\mathbb{T}^{d}\right)^{2}\right) \mapsto l_{2}\left(\left(\mathbb{Z}^{d}\right)^{2}\right)$ - стандартное преобразование Фурье, $L^{1}\left(\mathbb{T}^{d}\right)$ - банахово пространство интегрируемых функций на $\mathbb{T}^{d}$.

Двухчастичный гамильтониан в импульсном представлении определяется как ограниченный самосопряженный оператор $H_{\mu}=\widehat{\mathcal{F}}^{-1} \widehat{H}_{\mu} \widehat{\mathcal{F}} \equiv H_{0}+\mu V$, действующий в $L^{2}\left(\left(\mathbb{T}^{d}\right)^{2}\right)$. Двухчастичный свободный гамильтониан $H_{0}$ является оператором умножения на функцию:

$$
\left(H_{0} f\right)\left(k_{1}, k_{2}\right)=\left(\varepsilon\left(k_{1}\right)+\gamma \varepsilon\left(k_{2}\right)\right) f\left(k_{1}, k_{2}\right), \quad f \in L^{2}\left(\left(\mathbb{T}^{d}\right)^{2}\right),
$$

где

$$
\varepsilon(p)=\sum_{i=1}^{d}\left(1-\cos p^{(i)}\right), \quad p=\left(p^{(1)}, \ldots, p^{(d)}\right) \in \mathbb{T}^{d} .
$$

Возмущение $V$ является интегральным оператором в пространстве $L^{2}\left(\left(\mathbb{T}^{d}\right)^{2}\right)$ :

$$
(V f)\left(k_{1}, k_{2}\right)=\frac{1}{(2 \pi)^{d}} \int_{\left(\mathbb{T}^{d}\right)^{2}} \delta\left(k_{1}+k_{2}-k_{1}^{\prime}-k_{2}^{\prime}\right) f\left(k_{1}^{\prime}, k_{2}^{\prime}\right) d k_{1}^{\prime} d k_{2}^{\prime},
$$

где $\delta(k)-d$-мерная дельта-функция Дирака на $\mathbb{T}^{d}$. 
Пространство $L^{2}\left(\left(\mathbb{T}^{d}\right)^{2}\right)$ разлагается в прямой интеграл,

$$
L^{2}\left(\left(\mathbb{T}^{d}\right)^{2}\right)=\int_{K \in \mathbb{T}^{d}} \oplus L^{2}\left(\mathbb{T}^{d}\right) d K,
$$

следовательно, оператор $H_{\mu}$ также разлагается в прямой интеграл,

$$
H_{\mu}=\int_{K \in \mathbb{T}^{d}} \oplus H_{\mu}(K) d K,
$$

где слойный оператор $H_{\mu}(K), K \in \mathbb{T}^{d}$, действует в гильбертовом пространстве $L^{2}\left(\mathbb{T}^{d}\right)$ по формуле $H_{\mu}(K)=H_{0}(K)+\mu V$ [3]. Невозмущенный оператор $H_{0}(K)$ в гильбертовом пространстве $L^{2}\left(\mathbb{T}^{d}\right)$ есть оператор умножения на функцию $\mathcal{E}_{K}(\cdot)$ :

$$
\left(H_{0}(K) f\right)(q)=\mathcal{E}_{K}(q) f(q), \quad f \in L^{2}\left(\mathbb{T}^{d}\right),
$$

где

$$
\mathcal{E}_{K}(q)=\varepsilon(q)+\gamma \varepsilon(K-q),
$$

функция $\varepsilon(\cdot)$ определяется по формуле (1). Оператор взаимодействия (возмущение) $V$ действует в гильбертовом пространстве $L^{2}\left(\mathbb{T}^{d}\right)$ :

$$
(V f)(p)=\frac{1}{(2 \pi)^{d}} \int_{\mathbb{T}^{d}} f(q) d q, \quad f \in L^{2}\left(\mathbb{T}^{d}\right) .
$$

\section{3. ФОРМУЛИРОВКА ОСНОВНЫХ РЕЗУЛЬТАТОВ}

Возмущение $V$ оператора умножения $H_{0}(K)$ является одномерным ограниченным самосопряженным оператором. Из теоремы Вейля о сохранении существенного спектра при компактном возмущении следует, что существенный спектр оператора $H_{\mu}(K)$ совпадает со спектром оператора $H_{0}(K), K \in \mathbb{T}^{d}$, и представляет собой интервал:

$$
\sigma_{\mathrm{ess}}\left(H_{\mu}(K)\right)=\sigma\left(H_{0}(K)\right)=\left[\mathcal{E}_{\min }(K), \mathcal{E}_{\max }(K)\right]
$$

где

$$
\begin{aligned}
& \mathcal{E}_{\min }(K)=\min _{p \in \mathbb{T}^{d}} \mathcal{E}_{K}(p)=(1+\gamma) d-\sum_{i=1}^{d} \sqrt{1+2 \gamma \cos K^{(i)}+\gamma^{2}} \\
& \mathcal{E}_{\max }(K)=\max _{p \in \mathbb{T}^{d}} \mathcal{E}_{K}(p)=(1+\gamma) d+\sum_{i=1}^{d} \sqrt{1+2 \gamma \cos K^{(i)}+\gamma^{2}} .
\end{aligned}
$$

ЗАмЕчАНИЕ 1. В дальнейшем будем всюду предполагать, что $\gamma \neq 1$ и $d \geqslant 3$. При $\gamma=1$ и $K=\vec{\pi}=(\pi, \ldots, \pi) \in \mathbb{T}^{d}$ существенный спектр $\sigma_{\text {ess }}\left(H_{\mu}(K)\right)$ оператора $H_{\mu}(K)$ вырождается, т. е. превращается в точку $\mathcal{E}_{\min }(\vec{\pi})=\mathcal{E}_{\max }(\vec{\pi})=2 d$, и поэтому существенный спектр $\sigma_{\text {ess }}\left(H_{\mu}(K)\right)$ оператора $H_{\mu}(K)$ не является абсолютно непрерывным при всех $K \in \mathbb{T}^{d}$.

Пусть $\mathbb{C}$ - комплексная плоскость. Для любого $K \in \mathbb{T}^{d}$ определим аналитическую функцию $\nu(K, \cdot)$ в $\mathbb{C} \backslash\left[\mathcal{E}_{\min }(K), \mathcal{E}_{\max }(K)\right]$ по формуле

$$
\nu(K, z)=\frac{1}{(2 \pi)^{d}} \int_{\mathbb{T}^{d}} \frac{d q}{z-\mathcal{E}_{K}(q)} .
$$


Пусть $V^{1 / 2}$ - квадратный корень из положительного оператора $V$ и $G_{\mu}(K, z)-$ интегральный оператор Бирмана-Швингера ранга 1 в гильбертовом пространстве $L^{2}\left(\mathbb{T}^{d}\right)$ с ядром $\left(\mu /(2 \pi)^{d}\right) \nu(K, z), K \in \mathbb{T}^{d}, z>\mathcal{E}_{\max }(K)$. Следующее утверждение хорошо известный принцип Бирмана-Швингера - доказан для широкого класса операторов на решетке в работе [15].

Лемма 1. Пусть $z>\mathcal{E}_{\max }(K)$. Справедливы следующие утверждения:

1) если функиия $f \in L^{2}\left(\mathbb{T}^{d}\right)$ является решением уравнения $H_{\mu}(K) f=z f$, то функиия $\varphi=V^{1 / 2} f \in L^{2}\left(\mathbb{T}^{d}\right)$ является решением уравнения $\varphi=G_{\mu}(K, z) \varphi$;

2) если функция $\varphi \in L^{2}\left(\mathbb{T}^{d}\right)$ является решением уравнения $\varphi=G_{\mu}(K, z) \varphi$, то $f=\left[z-H_{0}(K)\right]^{-1} V^{1 / 2} \varphi \in L^{2}\left(\mathbb{T}^{d}\right)$ является решением уравнения $H_{\mu}(K) f=z f ;$

3) число $z$ является гда и только тогда, когда число 1 является оператора $G_{\mu}(K, z)$;

4) с учетом кратности число собственных значений оператора $H_{\mu}(K)$, менъших $z$, равно числу собственных значений оператора $G_{\mu}(K, z)$, больших 1.

Для любого $K \in \mathbb{T}^{d}$ функция $\mathcal{E}_{K}(\cdot)$ имеет невырожденный максимум в точке $p=\vec{\pi}+p(K)$, поэтому существует интеграл

$$
\int_{\mathbb{T}^{d}} \frac{d q}{\mathcal{E}_{\max }(K)-\mathcal{E}_{K}(q)}
$$

и определен предельный интегральный оператор Бирмана-Швингера

$$
G_{\mu}\left(K, \mathcal{E}_{\max }(K)\right)=\mu V^{1 / 2}\left(\mathcal{E}_{\max }(K)-H_{0}(K)\right)^{-1} V^{1 / 2}
$$

с ядром $\left(\mu /(2 \pi)^{d}\right) \nu\left(K, \mathcal{E}_{\max }(K)\right)$.

ЗАмечание 2 . Пусть $K \in \mathbb{T}^{d}$. Оператор $H_{\mu}(K)$ имеет собственное значение $z \geqslant \mathcal{E}_{\max }(K)$, т. е. $\operatorname{Ker}\left(H_{\mu}(K)-z I\right) \neq 0$, тогда и только тогда, когда компактный оператор $G_{\mu}(K, z)$, действующий в $L^{2}\left(\mathbb{T}^{d}\right)$, имеет собственное значение 1 и существует функция $\psi \in \operatorname{Ker}\left(G_{\mu}(K, z)-I\right)$ такая, что функция

$$
f(p)=\frac{\left(V^{1 / 2} \psi\right)(p)}{z-\mathcal{E}_{K}(p)}, \quad p \in \mathbb{T}^{d}
$$

принадлежит пространству $L^{2}\left(\mathbb{T}^{d}\right)$. В этом случае $f \in \operatorname{Ker}\left(H_{\mu}(K)-z I\right)$. Кроме того, если $z>\mathcal{E}_{\max }(K)$, то

$$
\operatorname{dim} \operatorname{Ker}\left(H_{\mu}(K)-z I\right)=\operatorname{dim} \operatorname{Ker}\left(G_{\mu}(K, z)-I\right)
$$

И

$$
\operatorname{Ker}\left(H_{\mu}(K)-z I\right)=\left\{f: f(\cdot)=\frac{\left(V^{1 / 2} \psi\right)(\cdot)}{z-\mathcal{E}_{K}(\cdot)}, \psi \in \operatorname{Ker}\left(G_{\mu}(K, z)-I\right)\right\} .
$$

ЗАмЕЧАниЕ 3 . Если $z=\mathcal{E}_{\max }(K)$, то равенство (5) может не выполняться. В этом случае его нужно заменить на следующее неравенство:

$$
\operatorname{dim} \operatorname{Ker}\left(H_{\mu}(K)-\mathcal{E}_{\max }(K) I\right) \leqslant \operatorname{dim} \operatorname{Ker}\left(G_{\mu}(K, z)-I\right) ;
$$

если $\psi$ является собственной функцией оператора $G_{\mu}\left(K, \mathcal{E}_{\max }(K)\right)$, соответствующей собственному значению 1 , то функция

$$
f(\cdot)=\frac{\mu\left(V^{1 / 2} \psi\right)(\cdot)}{\mathcal{E}_{\max }(K)-\mathcal{E}_{K}(\cdot)}
$$


является решением уравнения $H_{\mu}(K) f=\mathcal{E}_{\max }(K) f$. Однако данная функция не принадлежит пространству $L^{2}\left(\mathbb{T}^{d}\right)$, если $d=3,4$ и $\left(V^{1 / 2} \psi\right)(\vec{\pi}+p(K)) \neq 0$, где $p(K)=$ $\left(p\left(K^{(1)}\right), \ldots, p\left(K^{(d)}\right)\right)$ и

$$
p\left(K^{(i)}\right)=\arcsin \frac{\gamma \sin K^{(i)}}{\sqrt{1+2 \gamma \cos K^{(i)}+\gamma^{2}}}, \quad K^{(i)} \in \mathbb{T}^{1}, \quad i=1, \ldots, d .
$$

ОПРЕДЕЛЕНИЕ. Пусть $d=3,4$. Будем говорить, что оператор $H_{\mu}(K)$ имеет виртуальный уровень на правом краю (в точке $\left.z=\mathcal{E}_{\max }(K)\right)$ существенного спектра $\sigma_{\mathrm{ess}}\left(H_{\mu}(K)\right)$, если число 1 является простым собственным значением оператора

$$
G_{\mu}\left(K, \mathcal{E}_{\max }(K)\right)=\mu V^{1 / 2}\left(\mathcal{E}_{\max }(K)-H_{0}(K)\right)^{-1} V^{1 / 2}
$$

и соответствующая собственная функция $\psi$ удовлетворяет условию

$$
\left(V^{1 / 2} \psi\right)(\vec{\pi}+p(K)) \neq 0 .
$$

Мы всегда можем считать, что $\left(V^{1 / 2} \psi\right)(\vec{\pi}+p(K))=1$.

Введем обозначение $\nu(K)=\nu\left(K, \mathcal{E}_{\max }(K)\right)$ и для любого $\mu>0$ определим следующие множества:

$$
\begin{aligned}
& M_{<}(\mu)=\left\{K \in \mathbb{T}^{d}: 1-\mu \nu(K)<0\right\}, \\
& M_{=}(\mu)=\left\{K \in \mathbb{T}^{d}: 1-\mu \nu(K)=0\right\}, \\
& M_{>}(\mu)=\left\{K \in \mathbb{T}^{d}: 1-\mu \nu(K)>0\right\} .
\end{aligned}
$$

ЗАмЕЧАнИЕ 4. Функция $\nu(K)$ (см. лемму 2 ниже) является аналитической на торе $\mathbb{T}^{d}$, и поэтому подмножество $M_{=}(\mu)$ - многообразие коразмерности 1 в $\mathbb{T}^{d}$. Более того, $M_{<}(\mu)$ и $M_{>}(\mu)$ - открытые связные множества в $\mathbb{T}^{d}$.

Отметим (см. лемму 3 ниже), что в силу аналитичности функции $\nu(K)$ и компактности пространства $\mathbb{T}^{d}$ справедливы неравенства $0<\nu(0) \leqslant \nu(K) \leqslant \nu(\vec{\pi})$ при всех $K \in \mathbb{T}^{d}$.

Основные результаты настоящей работы формулируются в следующих теоремах.

Теорема 1. Имеют место следующие утверждения.

1. Пусть $0<\mu<1 / \nu(\vec{\pi})$, тогда $M_{<}(\mu)=\varnothing, M_{=}(\mu)=\varnothing u M_{>}(\mu)=\mathbb{T}^{d}$.

2. Пусть $\mu=1 / \nu(\vec{\pi})$, mогда $M_{<}(\mu)=\varnothing, M_{=}(\mu)=\{\vec{\pi}\}$ u $M_{>}(\mu)=\mathbb{T}^{d} \backslash\{\vec{\pi}\}$.

3. Пусть $1 / \nu(\vec{\pi})<\mu<1 / \nu(0)$, тогда каждое из множеств $M_{<}(\mu), M_{=}(\mu) u$ $M_{>}(\mu)$ является непустым.

4. Пусть $\mu=1 / \nu(0)$, тогда $M_{<}(\mu)=\mathbb{T}^{d} \backslash\{0\}, M_{=}(\mu)=\{0\} u M_{>}(\mu)=\varnothing$.

5. Пусть $\mu>1 / \nu(0)$, тогда $M_{<}(\mu)=\mathbb{T}^{d}, M_{=}(\mu)=\varnothing u M_{>}(\mu)=\varnothing$.

Teорема 2. 1. Пусть $\mu>0 u K \in M_{<}(\mu)$. Тогда оператор $H_{\mu}(K)$ имеет единственное собственное значение $E_{\mu}(K)$, лежащее правее существенного спектра $\sigma_{\mathrm{ess}}\left(H_{\mu}(K)\right)$. Соответствующее собственное состояние

$$
\psi_{\mu, K}(\cdot)=c \cdot \frac{\mu}{E_{\mu}(K)-\mathcal{E}_{K}(\cdot)},
$$

где с $\neq 0$ - нормировочный множитель, принадлежит пространству $L^{2}\left(\mathbb{T}^{d}\right)$ и является вещественно-аналитической функиией. Кроме того, $E_{\mu}(K)$ - четная вещественно-аналитическая функиия на $M_{<}(\mu)$. Отображение $\psi_{\mu}: M_{<}(\mu) \mapsto L^{2}\left(\mathbb{T}^{d}\right)$ 
такое, что $K \mapsto \psi_{\mu, K}(\cdot)$, является векторнозначным аналитическим отображением. Если $0<\mu \leqslant 1 / \nu(0)$, то для собственного значения $E_{\mu}(K), K \in M_{<}(\mu)$, выполняются неравенства $\mathcal{E}_{\max }(K)<E_{\mu}(K)<\mathcal{E}_{\max }(0) ;$ если $\mu>1 / \nu(0)>0$, то собственное значение $E_{\mu}(K), K \in M_{<}(\mu)$, удовлетворяет неравенствам

$$
\mathcal{E}_{\max }(0)<E_{\mu}(0), \quad \mathcal{E}_{\max }(K)<E_{\mu}(K)<E_{\mu}(0) .
$$

2. Пусть $\mu>0$ и $K \in M_{=}(\mu)$. При $d=3,4$ правый край $\mathcal{E}_{\max }(K)$ существенного спектра $\sigma_{\mathrm{ess}}\left(H_{\mu}(K)\right)$ является виртуальным уровнем оператора $H_{\mu}(K)$. Соответствующее виртуальное состояние

$$
\psi_{\mu, K}(\cdot)=c \cdot \frac{\mu}{\mathcal{E}_{\max }(K)-\mathcal{E}_{K}(\cdot)},
$$

где $c \neq 0$ - нормировочный множитель, принадлежит $L^{1}\left(\mathbb{T}^{d}\right) \backslash L^{2}\left(\mathbb{T}^{d}\right)$. При $d \geqslant 5$ u $K \in M_{=}(\mu)$ правый край $\mathcal{E}_{\max }(K)$ существенного спектра $\sigma_{\mathrm{ess}}\left(H_{\mu}(K)\right)$ является собственным значением оператора $H_{\mu}(K)$. Соответствующее собственное состояние имеет вид

$$
\psi_{\mu, K}(\cdot)=c \cdot \frac{\mu}{\mathcal{E}_{\max }(K)-\mathcal{E}_{K}(\cdot)},
$$

где $c \neq 0$ - нормировочный множитель, и принадлежит пространству $L^{2}\left(\mathbb{T}^{d}\right)$.

3. Пусть $\mu>0$ и $K \in M_{>}(\mu)$. Тогда оператор $H_{\mu}(K)$ не имеет собственного значения, лежащего правее существенного спектра $\sigma_{\mathrm{ess}}\left(H_{\mu}(K)\right)$.

ЗАмечАниЕ 5. Множество $M_{<}(\mu)$ возрастает (расширяется) от пустого множества до тора $\mathbb{T}^{d}$ при возрастании константы связи $\mu>0$. Множество пороговых значений квазиимпульса $M_{=}(\mu)$ характеризуется тем, что для любой точки $K_{0} \in M_{=}(\mu)$ и для любой ее окрестности $W\left(K_{0}\right) \subset \mathbb{T}^{d}$ найдутся точки $K_{0}^{\prime} \in M_{<}(\mu)$ и $K_{0}^{\prime \prime} \in M_{>}(\mu)$ такие, что число собственных значений операторов $H_{\mu}\left(K_{0}^{\prime}\right)$ и $H_{\mu}\left(K_{0}^{\prime \prime}\right)$ отличается на единицу.

ЗАмечАниЕ 6. Как следует из утверждения 2 теоремы 2, виртуальное состояние $\psi_{\mu, K}(\cdot)$, соответствующее пороговому виртуальному уровню, или пороговое собственное состояние, соответствующее пороговому собственному значению, не являются даже непрерывными функциями на $\mathbb{T}^{d}$. Однако собственное состояние, соответствующее собственному значению, которое лежит вне существенного спектра $\sigma_{\text {ess }}\left(H_{\mu}(K)\right)$ оператора $H_{\mu}(K)$, является аналитической функцией на $\mathbb{T}^{d}$.

ЗАмЕчАниЕ 7. Из свойств интеграла Лебега от неотрицательных функций следует, что множества $M_{<}(\mu), M_{=}(\mu)$ и $M_{>}(\mu)$ можно определить также следующим эквивалентным способом:

$$
\begin{aligned}
& M_{<}(\mu)=\left\{K \in \mathbb{T}^{d}: \mu>\mathcal{E}_{\max }(K)-\mathcal{E}_{K}(q), q \in \mathbb{T}^{d}\right\}, \\
& M_{=}(\mu)=\left\{K \in \mathbb{T}^{d}: \mu=\mathcal{E}_{\max }(K)-\mathcal{E}_{K}(q), q \in \mathbb{T}^{d}\right\}, \\
& M_{>}(\mu)=\left\{K \in \mathbb{T}^{d}: \mu<\mathcal{E}_{\max }(K)-\mathcal{E}_{K}(q), q \in \mathbb{T}^{d}\right\} .
\end{aligned}
$$

\section{4. ДОКАЗАТЕЛЬСТВА ОСНОВНЫХ РЕЗУЛЬТАТОВ}

Предварительно докажем несколько лемм.

Функцию $\mathcal{E}_{K}(p)$, определенную формулой $(2)$, можно представить в виде

$$
\mathcal{E}_{K}(p)=(1+\gamma) d-\sum_{i=1}^{d} \sqrt{1+2 \gamma \cos K^{(i)}+\gamma^{2}} \cos \left(p^{(i)}-p\left(K^{(i)}\right)\right),
$$


где $p\left(K^{(i)}\right)$ заданы в $(6)$. Положим

$$
a\left(K^{(i)}\right)=\sqrt{1+2 \gamma \cos K^{(i)}+\gamma^{2}} \geqslant 0 .
$$

Отсюда немедленно вытекает следующее утверждение.

ПРЕДЛОЖЕНИЕ 1. Справедливы следующие соотношения: для всех $i=1, \ldots, d$

$$
\begin{array}{ll}
a\left(K^{(i)}\right)=a\left(-K^{(i)}\right), & K^{(i)} \in \mathbb{T}^{1}, \\
a\left(K^{(i)}\right)>\min _{K^{(i)} \in \mathbb{T}^{1}} a\left(K^{(i)}\right)=|1-\gamma|, & K^{(i)} \neq \pi, \\
a\left(K^{(i)}\right)<\max _{K^{(i)} \in \mathbb{T}^{1}} a\left(K^{(i)}\right)=1+\gamma, & K^{(i)} \neq 0 .
\end{array}
$$

ПРЕДЛОЖЕНИЕ 2. Справедливы следующие соотношения:

$$
\begin{array}{ll}
\mathcal{E}_{\min }(K)>\min _{K \in \mathbb{T}^{d}} \mathcal{E}_{\min }(K)=\mathcal{E}_{\min }(0)=0, & K \neq 0, \\
\mathcal{E}_{\min }(K)<\max _{K \in \mathbb{T}^{d}} \mathcal{E}_{\min }(K)=\mathcal{E}_{\min }(\vec{\pi})=(1+\gamma-|1-\gamma|) d, & K \neq \vec{\pi}, \\
\mathcal{E}_{\max }(K)>\min _{K \in \mathbb{T}^{d}} \mathcal{E}_{\max }(K)=(1+\gamma+|1-\gamma|) d, & K \neq \vec{\pi}, \\
\mathcal{E}_{\max }(K)<\max _{K \in \mathbb{T}^{d}} \mathcal{E}_{\max }(K)=\mathcal{E}_{\max }(0)=2(1+\gamma) d, & K \neq 0 .
\end{array}
$$

Кроме того, при $\gamma \rightarrow 0$

$$
\mathcal{E}_{\min }(K) \rightarrow 0, \quad \mathcal{E}_{\max }(K) \rightarrow 2 d .
$$

ДокАзАтельство. Опираясь на утверждения (9б) и (9в) предложения 1 , получим соотношения (10a) и (10б): при $K \neq 0$

$$
\begin{aligned}
\mathcal{E}_{\min }(K) & =(1+\gamma) d-\sum_{i=1}^{d} a\left(K^{(i)}\right)>\min _{K \in \mathbb{T}^{d}}\left[(1+\gamma) d-\sum_{i=1}^{d} a\left(K^{(i)}\right)\right]= \\
& =(1+\gamma) d-\sum_{i=1}^{d} \max _{K^{(i)}} a\left(K^{(i)}\right)=(1+\gamma) d-\sum_{i=1}^{d}(1+\gamma)=0
\end{aligned}
$$

при $K \neq \vec{\pi}$

$$
\begin{aligned}
\mathcal{E}_{\min }(K) & =(1+\gamma) d-\sum_{i=1}^{d} a\left(K^{(i)}\right)<\max _{K \in \mathbb{T}^{d}}\left[(1+\gamma) d-\sum_{i=1}^{d} a\left(K^{(i)}\right)\right]= \\
& =(1+\gamma) d-\sum_{i=1}^{d} \min _{K^{(i)}} a\left(K^{(i)}\right)=(1+\gamma) d-\sum_{i=1}^{d}|1-\gamma|= \\
& =(1+\gamma-|1-\gamma|) d .
\end{aligned}
$$

Формулы (10в) и (10г) доказываются аналогично. Утверждение (11) вытекает из формул (3) для $\mathcal{E}_{\max }(K)$ и $\mathcal{E}_{\min }(K)$.

Лемма 2. Пусть $K \in \mathbb{T}^{d}$. Тогда существует предел $\lim _{z \rightarrow \mathcal{E}_{\max }(K)} \nu(K, z)<+\infty$. Более того, равенство

$$
\nu(K)=\nu\left(K, \mathcal{E}_{\max }(K)\right)=\frac{1}{(2 \pi)^{d}} \int_{\mathbb{T}^{d}} \frac{d q}{\mathcal{E}_{\max }(K)-\mathcal{E}_{K}(q)}
$$

задает аналитическую функцию на торе $\mathbb{T}^{d}$. 
ДокАзАтЕЛЬСтво. Из равенства $(7)$ следует, что функция $\mathcal{E}_{K}(\cdot)$ имеет невырожденный максимум в точке $p:=\vec{\pi}+p(K)$. Функцию $\nu(K, z)$, определенную по формуле (4), представим в виде

$$
\nu(K, z)=\frac{1}{(2 \pi)^{d}} \int_{\mathbb{T}^{d}} \frac{d p}{z-\mathcal{E}_{\max }(K)+\sum_{i=1}^{d} a\left(K^{(i)}\right)\left[1+\cos \left(p^{(i)}-p\left(K^{(i)}\right)\right)\right]} .
$$

Заменяя в этом интеграле переменную $p:=q+p(K)$ на $q$, получим

$$
\nu(K, z)=\frac{1}{(2 \pi)^{d}} \int_{\mathbb{T}^{d}} \frac{d q}{z-\mathcal{E}_{\max }(K)+\sum_{i=1}^{d} a\left(K^{(i)}\right)\left(1+\cos q^{(i)}\right)},
$$

при этом точка $q=\vec{\pi}$ является единственной невырожденной точкой минимума функции $\sum_{i=1}^{d} a\left(K^{(i)}\right)\left(1+\cos q^{(i)}\right)$.

Пусть $W_{\theta}(0)$ - шар достаточно малого радиуса $\theta>0$ с центром в точке $y=0$. Рассмотрим отображение $\phi_{K}: W_{\theta}(0) \mapsto \widetilde{W}(\vec{\pi})$ такое, что

$$
q^{(i)} \mapsto 2 \arccos \frac{y_{i}}{\sqrt{2 a\left(K^{(i)}\right)}}, \quad K^{(i)} \in \mathbb{T}^{1}, \quad i=1, \ldots, d,
$$

где $\widetilde{W}(\vec{\pi}):=\phi_{K}\left(W_{\theta}(0)\right)$ - образ шара $W_{\theta}(0)$. Функцию $\nu(K, z)$ представим в виде суммы двух функций:

$$
\begin{aligned}
\nu(K, z)= & \frac{1}{(2 \pi)^{d}} \int_{\widetilde{W}(\vec{\pi})} \frac{d q}{z-\mathcal{E}_{\max }(K)+\sum_{i=1}^{d} a\left(K^{(i)}\right)\left(1+\cos q^{(i)}\right)}+ \\
& \quad+\frac{1}{(2 \pi)^{d}} \int_{\mathbb{T}^{d} \backslash \widetilde{W}(\vec{\pi})} \frac{d q}{z-\mathcal{E}_{\max }(K)+\sum_{i=1}^{d} a\left(K^{(i)}\right)\left(1+\cos q^{(i)}\right)}= \\
= & \nu_{1}(K, z)+\nu_{2}(K, z) .
\end{aligned}
$$

При всех $K \in \mathbb{T}^{d}$ и $z \geqslant \mathcal{E}_{\max }(K)$ функция

$$
z-\mathcal{E}_{\max }(K)+\sum_{i=1}^{d} a\left(K^{(i)}\right)\left(1+\cos q^{(i)}\right)
$$

в замкнутой области $\mathbb{T}^{d} \backslash \widetilde{W}(\vec{\pi})$ строго положительна, поэтому функции $\nu_{2}(\cdot, z)$ при $z \geqslant \mathcal{E}_{\max }(\cdot)$ и $\nu_{2}(K, \cdot)$ при $K \in \mathbb{T}^{d}$ являются вещественно-аналитическими функциями на торе $\mathbb{T}^{d}$ и на полубесконечном интервале $\left(\mathcal{E}_{\max }(\cdot),+\infty\right)$ соответственно. В первом интеграле равенства (12), заменяя переменную $q:=\phi_{K}(y)$ на $y$, получим

$$
\nu_{1}(K, z)=\int_{W_{\theta}(0)} \frac{J\left(\phi_{K}(y), K\right)}{z-\mathcal{E}_{\max }(K)+\sum_{i=1}^{d} y_{i}^{2}} d y .
$$

Якобиан $J\left(\phi_{K}(y), K\right)$ преобразования $\phi_{K}(y)$ равен

$$
J\left(\phi_{K}(y), K\right)=2^{d} \prod_{i=1}^{d} \frac{1}{\sqrt{2 a\left(K^{(i)}\right)-y_{i}^{2}}} .
$$

Перейдем к сферическим координатам $y=r \omega$ в интеграле $(13)$, получим

$$
\nu_{1}(K, z)=\int_{0}^{\theta} \frac{r^{d-1}}{z-\mathcal{E}_{\max }(K)+r^{2}}\left\{\int_{\Omega_{d-1}} J\left(\phi_{K}(r \omega), K\right) d \omega\right\} d r,
$$


где $\Omega_{d-1}$ - единичная сфера в $\mathbb{R}^{d-1}$ и $d \omega$ - элемент площади единичной сферы. При $d \geqslant 3$, используя соотношение (14), имеем

$$
\nu_{1}\left(K, \mathcal{E}_{\max }(K)\right)=\int_{0}^{\theta} r^{d-3}\left\{\int_{\Omega_{d-1}} J\left(\phi_{K}(r \omega), K\right) d \omega\right\} d r,
$$

где $J\left(\phi_{(\cdot)}(r \omega), \cdot\right)$ - аналитическая функция на $\mathbb{T}^{d}$. Следовательно, $\nu_{1}\left(\cdot, \mathcal{E}_{\max }(\cdot)\right)$ является аналитической функцией на $\mathbb{T}^{d}$.

СледСтвиЕ. Пусть $K \in \mathbb{T}^{d}$. Eсли $d=3,4$, то функиия

$$
f(\cdot)=\frac{\mu\left(V^{1 / 2} \psi\right)(\cdot)}{\mathcal{E}_{\max }(K)-\mathcal{E}_{K}(\cdot)}
$$

принадлежит $L^{1}\left(\mathbb{T}^{d}\right) \backslash L^{2}\left(\mathbb{T}^{d}\right)$. Если $d \geqslant 5$, то эта функция принадлежит пространству $L^{2}\left(\mathbb{T}^{d}\right)$.

Для доказательства достаточно заметить, что $\left(V^{1 / 2} \psi\right)(p)=c \neq 0$ и воспользоваться доказательством леммы 2.

Лемма 3. 1. Функиия $\nu(K)=\nu\left(K_{1}, \ldots, K_{d}\right)$ является четной на $\mathbb{T}^{d}$ и для любого фиксированного $\left(K^{(1)}, \ldots, K^{(i-1)}, K^{(i+1)}, \ldots, K^{(d)}\right) \in \mathbb{T}^{d-1}$ монотонно возрастает (монотонно убъвает) при $K^{(i)} \in(0, \pi)$ (соответственно при $K^{(i)} \in(-\pi, 0)$ ) для всех $i=1, \ldots, d$.

2. Пусть $K \in \mathbb{T}^{d}$. Тогда именот место соотношения

$$
\min _{K \in \mathbb{T}^{d}} \nu(K)=\nu(0) \leqslant \nu(K) \leqslant \nu(\vec{\pi})=\max _{K \in \mathbb{T}^{d}} \nu(K) .
$$

ДокАзАТЕльство. Первое утверждение вытекает из следующего представления функции $\nu(K)$ :

$$
\nu(K)=\nu\left(K, \mathcal{E}_{\max }(K)\right)=\frac{1}{(2 \pi)^{d}} \int_{\mathbb{T}^{d}} \frac{d q}{\sum_{i=1}^{d} a\left(K^{(i)}\right)\left(1+\cos q^{(i)}\right)} .
$$

В самом деле, $a_{\gamma}\left(K^{(i)}\right)$ - четная функция переменной $K^{(i)} \in \mathbb{T}^{1}, i=1, \ldots, d$, следовательно, функция $\nu(K)$ является четной. Функция $a\left(K^{(i)}\right), K^{(i)} \in \mathbb{T}^{1}, i=1, \ldots, d$, монотонно убывает (монотонно возрастает) на интервале $(0, \pi)$ (соответственно на интервале $(-\pi, 0))$. Из монотонности интеграла Лебега вытекает, что для любого фиксированного $\left(K^{(1)}, \ldots, K^{(i-1)}, K^{(i+1)}, \ldots, K^{(d)}\right) \in \mathbb{T}^{d-1}$ функция $\nu(K)$ монотонно возрастает (монотонно убывает) на интервале $(0, \pi)$ (соответственно на интервале $(-\pi, 0))$.

2. В силу предложения 1 верны следующие соотношения:

$$
\begin{aligned}
\nu(K) & =\frac{1}{(2 \pi)^{d}} \int_{\mathbb{T}^{d}} \frac{d q}{\sum_{i=1}^{d} \sqrt{1+2 \gamma \cos K^{(i)}+\gamma^{2}}\left(1+\cos q^{(i)}\right)} \geqslant \\
& \geqslant \frac{1}{(2 \pi)^{d}} \int_{\mathbb{T}^{d}} \frac{d q}{\sum_{i=1}^{d} \max _{K} a\left(K^{(i)}\right)\left(1+\cos q^{(i)}\right)}= \\
& =\frac{1}{(2 \pi)^{d}} \frac{1}{1+\gamma} \int_{\mathbb{T}^{d}} \frac{d q}{\sum_{i=1}^{d}\left(1+\cos q^{(i)}\right)}=\nu(0),
\end{aligned}
$$




$$
\begin{aligned}
\nu(K) & \leqslant \frac{1}{(2 \pi)^{d}} \int_{\mathbb{T}^{d}} \frac{d q}{\sum_{i=1}^{d} \min _{K} a\left(K^{(i)}\right)\left(1+\cos q^{(i)}\right)}= \\
& =\frac{1}{(2 \pi)^{d}} \frac{1}{|1-\gamma|} \int_{\mathbb{T}^{d}} \frac{d q}{\sum_{i=1}^{d}\left(1+\cos q^{(i)}\right)}=\nu(\vec{\pi}) .
\end{aligned}
$$

Лемма доказана.

Для любых $K \in \mathbb{T}^{d}$ и $z \in \mathbb{C} \backslash\left[\mathcal{E}_{\min }(K), \mathcal{E}_{\max }(K)\right]$ определитель Фредгольма, ассоциированный с оператором $H_{\mu}(K)$, равен $\Delta_{\mu}(K, z)=1-\mu \nu(K, z)$. Заметим, что $\Delta_{\mu}(K, z)$ - вещественно-аналитическая функция в $\mathbb{T}^{d} \times\left(\mathbb{C} \backslash\left[\mathcal{E}_{\min }(K), \mathcal{E}_{\max }(K)\right]\right)$.

Следующая лемма является простым следствием принципа Бирмана-Швингера и теоремы Фредгольма. Мы приведем ее доказательство, чтобы установить общий вид собственной функции.

Лемма 4. Пусть $K \in \mathbb{T}^{d}$. Точка $z \in \mathbb{C} \backslash \sigma_{\mathrm{ess}}\left(H_{\mu}(K)\right)$ является собственным значением оператора $H_{\mu}(K)$ тогда и только тогда, когда $\Delta_{\mu}(K, z)=0$.

ДоказАтельство. Пусть точка $z=E_{\mu}(K) \in \mathbb{C} \backslash\left[\mathcal{E}_{\min }(K), \mathcal{E}_{\max }(K)\right]$ является собственным значением оператора $H_{\mu}(K), K \in \mathbb{T}^{d}$, и $0 \neq \psi \in L^{2}\left(\mathbb{T}^{d}\right)$ - соответствующая собственная функция, т. е.

$$
\left(E_{\mu}(K)-\mathcal{E}_{K}(q)\right) \psi(q)=\frac{\mu}{(2 \pi)^{d}} \int_{\mathbb{T}^{d}} \psi(t) d t .
$$

Отсюда заключаем, что собственная функция $\psi(\cdot) \equiv \psi_{\mu, K}(\cdot)$ имеет вид

$$
\psi_{\mu, K}(q)=c \cdot \frac{\mu}{E_{\mu}(K)-\mathcal{E}_{K}(q)},
$$

где $c \neq 0$ - нормировочный множитель. Проинтегрировав и умножив на $(2 \pi)^{-d}$ обе части последнего равенства, получим

$$
c=\frac{1}{(2 \pi)^{d}} \int_{\mathbb{T}^{d}} \psi_{\mu, K}(t) d t=\frac{c \mu}{(2 \pi)^{d}} \int_{\mathbb{T}^{d}} \frac{d t}{z-\mathcal{E}_{K}(t)},
$$

таким образом, $c \Delta_{\mu}(K, z)=0$.

Пусть уравнение $\Delta_{\mu}(K, z)=0$ имеет решение $z=E_{\mu}(K) \in \mathbb{C} \backslash\left[\mathcal{E}_{\min }(K), \mathcal{E}_{\max }(K)\right]$, тогда для любого $c \neq 0$ функция $\psi$, определенная по формуле (15), принадлежит пространству $L^{2}\left(\mathbb{T}^{d}\right)$ и удовлетворяет уравнению $H_{\mu}(K) \psi=z \psi$.

ПреДЛОЖЕНИЕ 3. 1. Подмножество $M_{<}(\mu)$ (подмножество $\left.M_{>}(\mu)\right)$ cuмметрично, т.е. если $K \in M_{<}(\mu)$ (соответственно $\left.K \in M_{>}(\mu)\right)$, то $-K \in M_{<}(\mu)$ (соответственно $\left.-K \in M_{>}(\mu)\right)$.

2. Если $\mu^{\prime}<\mu^{\prime \prime}$, mo $M_{<}\left(\mu^{\prime}\right) \subset M_{<}\left(\mu^{\prime \prime}\right)$.

ДокАзАтельство. Первое утверждение вытекает из четности функции $\nu(\cdot)$ на торе $\mathbb{T}^{d}$. Докажем второе утверждение.

Пусть $\mu^{\prime}<\mu^{\prime \prime}$ и $K \in M_{<}\left(\mu^{\prime}\right)$. Функция $1-\mu \nu(K)$ как функция переменной $\mu$ монотонно убывает, поэтому из неравенства $1-\mu^{\prime} \nu(K)<0$ следует неравенство $1-\mu^{\prime \prime} \nu(K)<0$, т. е. $M_{<}\left(\mu^{\prime}\right) \subset M_{<}\left(\mu^{\prime \prime}\right)$. 
Лемма 5. Пусть $d=3,4 u K \in \mathbb{T}^{d}$. Следующие утвержсдения эквивалентны:

1) число $z=\mathcal{E}_{\max }(K)$ является виртуальным уровнем оператора $H_{\mu}(K)$, и соответствующее виртуальное состояние имеет вид

$$
\psi_{\mu, K}(\cdot)=c \cdot \frac{\mu}{\mathcal{E}_{\max }(K)-\mathcal{E}_{K}(\cdot)} \in L^{1}\left(\mathbb{T}^{d}\right) \backslash L^{2}\left(\mathbb{T}^{d}\right),
$$

где $c \neq 0$ - нормировочный множитель;

2) $\Delta_{\mu}\left(K, \mathcal{E}_{\max }(K)\right)=1-\mu \nu(K)=0$;

3) $\mu=1 / \nu(K)$.

ДоказАтеЛьство. Достаточно доказать, что $1 \Rightarrow 2 \Rightarrow 3 \Rightarrow 1$.

Пусть $d=3,4$ и число $z=\mathcal{E}_{\max }(K)$ - виртуальный уровень оператора $H_{\mu}(K)$. Согласно определению виртуального уровня (см. раздел 3) число 1 является собственным значением оператора $G_{\mu}\left(K, \mathcal{E}_{\max }(K)\right)$, т. е. уравнение

$$
\varphi(p)=\frac{\mu}{(2 \pi)^{d}} \int_{\mathbb{T}^{d}} \frac{1}{(2 \pi)^{d}} \int_{\mathbb{T}^{d}} \frac{d t}{\mathcal{E}_{\max }(K)-\mathcal{E}_{K}(t)} \varphi(q) d q
$$

имеет ненулевое решение. Единственная функция $\varphi(p)=c \neq 0$ является решением этого уравнения, поэтому из (16) следует равенство

$$
c\left(1-\frac{\mu}{(2 \pi)^{d}} \int_{\mathbb{T}^{d}} \frac{d q}{\mathcal{E}_{\max }(K)-\mathcal{E}_{K}(q)}\right)=0 .
$$

Таким образом,

$$
\Delta_{\mu}\left(K, \mathcal{E}_{\max }(K)\right)=1-\frac{\mu}{(2 \pi)^{d}} \int_{\mathbb{T}^{d}} \frac{d q}{\mathcal{E}_{\max }(K)-\mathcal{E}_{K}(q)}=0,
$$

т. е. $\mu=1 / \nu(K)$.

Пусть $\mu=1 / \nu(K)$. Тогда $\Delta_{\mu}\left(K, \mathcal{E}_{\max }(K)\right)=0$, и $\varphi(p)=c \neq 0 \in L^{2}\left(\mathbb{T}^{d}\right)$ является нетривиальным решением уравнения $G_{\mu}\left(K, \mathcal{E}_{\max }(K)\right) \varphi=\varphi$. По определению число $z=\mathcal{E}_{\max }(K)$ есть виртуальный уровень оператора $H_{\mu}(K)$, и соответствующее виртуальное состояние

$$
\psi_{\mu, K}(\cdot)=c \cdot \frac{\mu}{\mathcal{E}_{\max }(K)-\mathcal{E}_{K}(\cdot)}
$$

согласно следствию из леммы 2 принадлежит $L^{1}\left(\mathbb{T}^{d}\right) \backslash L^{2}\left(\mathbb{T}^{d}\right)$.

Аналогично предыдущей доказывается следующая лемма.

Лемма 6. Пусть $d \geqslant 5$ и $K \in \mathbb{T}^{d}$. Следующие утверждения эквивалентны:

1) число $z=\mathcal{E}_{\max }(K)$ является собственным значением оператора $H_{\mu}(K), u$ соответствующее собственное состояние имеет вид

$$
\psi_{\mu, K}(\cdot)=c \cdot \frac{\mu}{\mathcal{E}_{\max }(K)-\mathcal{E}_{K}(\cdot)} \in L^{2}\left(\mathbb{T}^{d}\right),
$$

где с - нормировочный множитель;

2) $\Delta_{\mu}\left(K, \mathcal{E}_{\max }(K)\right)=1-\mu \nu(K)=0$;

3) $\mu=1 / \nu(K)$.

Лемма 7. 1. При всех $K \in \mathbb{T}^{d} \backslash\{0\}$ и $z \geqslant \mathcal{E}_{\max }(0)$ имеет место неравенство $\nu(0, z)>\nu(K, z)$.

2. Пусть $\mu=1 / \nu(0)$. Тогда $\Delta_{\mu}\left(K, \mathcal{E}_{\max }(0)\right)>0$ для любого $K \in \mathbb{T}^{d} \backslash\{0\}$. 
ДокАЗАТЕЛьСтво. 1. Из определения $\nu(K, z)$ получим, что

$$
\begin{aligned}
\nu(0, z)-\nu(K, z) & =\frac{1}{(2 \pi)^{d}} \int_{\mathbb{T}^{d}}\left(\frac{1}{z-\mathcal{E}_{0}(q)}-\frac{1}{z-\mathcal{E}_{K}(q)}\right) d q= \\
& =\frac{1}{(2 \pi)^{d}} \int_{\mathbb{T}^{d}}\left(\frac{1}{z-(1+\gamma) \varepsilon(q)}-\frac{1}{z-(\varepsilon(q)+\gamma \varepsilon(K-q))}\right) d q= \\
& =\frac{1}{(2 \pi)^{d}} \int_{\mathbb{T}^{d}} \frac{\gamma[\varepsilon(q)-\varepsilon(K-q)]}{[z-(1+\gamma) \varepsilon(q)][z-(\varepsilon(q)+\gamma \varepsilon(K-q))]} d q .
\end{aligned}
$$

Заменяя переменную $q:=K / 2-p$ на $p$ и учитывая равенство $\nu(-K, z)=\nu(K, z)$, представим разность $\nu(0, z)-\nu(K, z)$ в следующем виде:

$$
\begin{aligned}
\nu(0, z)-\nu(K, z) & =\frac{\nu(0, z)-\nu(-K, z)+\nu(0, z)-\nu(K, z)}{2}= \\
& =\frac{1}{(2 \pi)^{d}} \int_{\mathbb{T}^{d}} \gamma\left[\varepsilon\left(\frac{K}{2}-p\right)-\varepsilon\left(\frac{K}{2}+p\right)\right]^{2} F_{z}(K, p) Q_{z}(K, p) d p,
\end{aligned}
$$

где

$$
\begin{aligned}
F_{z}(K, p) & =\frac{2 z-\mathcal{E}_{K}(K / 2-p)-\mathcal{E}_{K}(K / 2+p)}{\left[z-\mathcal{E}_{K}(K / 2-p)\right]\left[z-\mathcal{E}_{K}(K / 2+p)\right]}>0, \\
Q_{z}(K, p) & =\frac{1}{[z-(1+\gamma) \varepsilon(K / 2-p)][z-(1+\gamma) \varepsilon(K / 2+p)]}>0 .
\end{aligned}
$$

Таким образом, при всех $K \in \mathbb{T}^{d} \backslash\{0\}$ и $z \geqslant \mathcal{E}_{\max }(0)$ неравенство $\nu(0, z)-\nu(K, z)>0$ доказано.

2. Пусть $\mu=1 / \nu(0)$. Тогда в силу лемм 5 и 6 получим, что $\Delta_{\mu}\left(0, \mathcal{E}_{\max }(0)\right)=0$. Из первого утверждения доказываемой леммы следует, что

$$
\nu\left(0, \mathcal{E}_{\max }(0)\right)>\nu\left(K, \mathcal{E}_{\max }(0)\right),
$$

отсюда

$$
\Delta_{\mu}\left(K, \mathcal{E}_{\max }(0)\right)=1-\mu \nu\left(K, \mathcal{E}_{\max }(0)\right)>1-\mu \nu\left(0, \mathcal{E}_{\max }(0)\right)=0 .
$$

Таким образом, при всех $K \in \mathbb{T}^{d} \backslash\{0\}$ неравенство $\Delta_{\mu}\left(K, \mathcal{E}_{\max }(0)\right)>0$ доказано.

ДоказАтельство теоремы 1. Докажем утверждение 1 . Пусть $0<\mu<1 / \nu(\vec{\pi})$. Тогда в силу второго утверждения леммы 3 получим следующие соотношения:

$$
1-\mu \nu_{\gamma}(K)>1-\frac{\nu_{\gamma}(K)}{\nu(\vec{\pi})} \geqslant 0, \quad K \in \mathbb{T}^{d} .
$$

Следовательно, $M_{<}(\mu)=\varnothing$ и $M_{=}(\mu)=\varnothing$ и $M_{>}(\mu)=\mathbb{T}^{d}$.

Доказательство утверждений 2 и 4 теоремы также вытекает из второго утверждения леммы 3.

Докажем утверждение 3 теоремы. Пусть $1 / \nu(\vec{\pi})<\mu<1 / \nu(0)$. Тогда из непрерывности функции $\nu(K)$ следует существование таких $K^{\prime}, K^{\prime \prime}, K_{0} \in \mathbb{T}^{d} \backslash\{0, \vec{\pi}\}$, что $1-\mu \nu\left(K^{\prime}\right)<0,1-\mu \nu\left(K^{\prime \prime}\right)>0$ и $1-\mu \nu\left(K_{0}\right)=0$. Следовательно, каждое из множеств $M_{<}(\mu), M_{>}(\mu)$ и $M_{=}(\mu)$ является непустым. Из непрерывности функции $\nu(K)$ следует, что $M_{<}(\mu)$ и $M_{>}(\mu)$ - открытые связные подмножества тора $\mathbb{T}^{d}$. Из второго утверждения леммы 3 вытекает, что если множество $M_{>}(\mu)$ непусто, то $K=0 \in M_{>}(\mu)$.

Утверждение 5 теоремы доказывается аналогично утверждению 1 . Теорема 1 полностью доказана. 
ДоКАЗАТЕЛЬСТво теОРЕМЫ 2. Докажем утверждение 1.

Пусть $\mu>0$ и $K \in M_{<}(\mu)$. Тогда из определения множества $M_{<}(\mu)$ получим, что $1-\mu \nu(K)<0$, т. е. выполняется неравенство $\Delta_{\mu}\left(K, \mathcal{E}_{\max }(K)\right)<0$. В силу непрерывности и монотонности функции $\Delta_{\mu}(K, \cdot)$ существует единственное число $E_{\mu}(K) \in\left(\mathcal{E}_{\max }(K),+\infty\right)$ такое, что $\Delta_{\mu}\left(K, E_{\mu}(K)\right)=0$. В силу леммы 4 оператор $H_{\mu}(K)$ имеет собственное значение на интервале $\left(\mathcal{E}_{\max }(K),+\infty\right)$. Соответствующее собственное состояние определяется по формуле (15).

Функции $\Delta_{\mu}(\cdot, z)$ при $z \in \mathbb{C} \backslash\left[\mathcal{E}_{\min }(K), \mathcal{E}_{\max }(K)\right]$ и $\Delta_{\mu}(K, \cdot)$ при $K \in \mathbb{T}^{d}$ являются вещественно-аналитическими функциями на $\mathbb{T}^{d}$ и $\mathbb{C} \backslash\left[\mathcal{E}_{\min }(K), \mathcal{E}_{\max }(K)\right]$ соответственно. Уравнение $\Delta_{\mu}(K, z)=0$ имеет единственное решение $E_{\mu}(K)$. Следовательно, в силу теоремы о неявной функции $E_{\mu}(\cdot)$ является аналитической функцией на торе $\mathbb{T}^{d}$. Функция $\mathcal{E}_{K}(\cdot)$ аналитическая, причем $E_{\mu}(K)-\mathcal{E}_{K}(\cdot) \neq 0$ на $\mathbb{T}^{d}$. Следовательно, собственное состояние (15) есть аналитическая функция на $\mathbb{T}^{d}$. Аналитичность отображения $\psi_{\mu}$ вытекает из аналитичности вектор-функции

$$
\psi_{\mu, K}(q)=c \cdot \frac{\mu}{E_{\mu}(K)-\mathcal{E}_{K}(q)}
$$

как функции двух переменных $K, q \in \mathbb{T}^{d}$.

Очевидно, что для любых $K \in \mathbb{T}^{d}$ и $z \in\left(\mathcal{E}_{\max }(K),+\infty\right)$ имеет место равенство $\Delta_{\mu}(-K, z)=\Delta_{\mu}(K, z)$. Отсюда и из единственности решения уравнения $\Delta_{\mu}(K, z)$ следует, что $\Delta_{\mu}\left(-K, E_{\mu}(-K)\right)=\Delta_{\mu}\left(K, E_{\mu}(K)\right)=0$, и это доказывает четность $E_{\mu}(\cdot)$ как функции $K \in M_{<}(\mu)$.

В силу утверждения 2 леммы 7 при всех $K \neq 0$ и $\mu=1 / \nu(0)$ выполняется неравенство $\Delta_{\mu}\left(K, \mathcal{E}_{\max }(0)\right)>0$. Функция $\Delta_{\mu}(K, z)$ монотонно убывает по переменной $\mu>0$, поэтому при всех $0<\mu \leqslant 1 / \nu(0)$ выполняется неравенство $\Delta_{\mu}\left(K, \mathcal{E}_{\max }(0)\right)>0$. Для любого $K \in M_{<}(\mu)$ функция $\Delta_{\mu}(K, \cdot)$ монотонно возрастает на интервале $\left(\mathcal{E}_{\max }(K),+\infty\right)$, и выполняются соотношения

$$
\Delta_{\mu}\left(K, \mathcal{E}_{\max }(K)\right)<\Delta_{\mu}\left(K, E_{\mu}(K)\right)=0<\Delta_{\mu}\left(K, \mathcal{E}_{\max }(0)\right) .
$$

Следовательно, при $\mu \leqslant 1 / \nu(0)$ и $K \in M_{<}(\mu)$ собственное значение $E_{\mu}(K)$ оператора $H_{\mu}(K)$ лежит в интервале $\left(\mathcal{E}_{\max }(K), \mathcal{E}_{\max }(0)\right)$.

Пусть $\mu>1 / \nu(0)$. Из утверждения 1 леммы 7 при всех $K \in \mathbb{T}^{d} \backslash\{0\}$ и $z \geqslant \mathcal{E}_{\max }(0)$ получим, что $\Delta_{\mu}(0, z)<\Delta_{\mu}(K, z)$. Для любого $K \in \mathbb{T}^{d}$ функция $\Delta_{\mu}(K, \cdot)$ монотонно возрастает, и имеют место соотношения

$$
\Delta_{\mu}\left(K, E_{\mu}(K)\right)=0=\Delta_{\mu}\left(0, E_{\mu}(0)\right)<\Delta_{\mu}\left(K, E_{\mu}(0)\right) .
$$

Следовательно, $\mathcal{E}_{\max }(K)<E_{\mu}(K)<E_{\mu}(0)$ при всех ненулевых $K \in M_{<}(\mu)$.

Докажем утверждение 2 теоремы. Пусть $\mu>0$ и $K \in M_{=}(\mu)$. В силу лемм 5 и 6 с учетом определения множества $M_{=}(\mu)$ имеем, что при $d=3,4$ правый край $z=\mathcal{E}_{\max }(K)$ существенного спектра $\sigma_{\text {ess }}\left(H_{\mu}(K)\right)$ является виртуальным уровнем, а при $d \geqslant 5$ - собственным значением оператора $H_{\mu}(K)$.

Докажем утверждение 3 теоремы. Пусть $\mu>0$ и $K \in M_{>}(\mu)$. Тогда по определению множества $M_{>}(\mu)$ получим, что $1-\mu \nu(K)>0$, т. е. выполняется неравенство $\Delta_{\mu}\left(K, \mathcal{E}_{\max }(K)\right)>0$. Следовательно, функция $\Delta_{\mu}(K, \cdot)$ не имеет нулей на множестве $\left(\mathcal{E}_{\max }(K),+\infty\right)$, поэтому оператор $H_{\mu}(K)$ не имеет собственного значения, лежащего правее существенного спектра $\sigma_{\text {ess }}\left(H_{\mu}(K)\right)$. Теорема 2 полностью доказана. 
Благодарности. Авторы приносят глубокую благодарность Р. А. Минлосу за ценные советы. Работа поддержана Фондом фундаментальных исследований Республики Узбекистан.

\section{Список литературы}

[1] A. I. Mogilner, "Hamiltonians in solid-state physics as multiparticle discrete Schrodinger operators: problems and results", Many Particle Hamiltonians: Spectra and Scattering, Advances in Soviet Mathematics, 5, ed. R. A. Minlos, AMS, Providence, RI, 1991, 139-194.

[2] D. C. Mattis, Rev. Mod. Phys., 58:2 (1986), 361-379.

[3] S. Albeverio, S. N. Lakaev, K. A. Makarov, Z. I. Muminov, Commun. Math. Phys., 262:1 (2006), 91-115, arXiv: math-ph/0501013.

[4] S. Albeverio, F. Gesztesy, R. Høegh-Krohn, Ann. Inst. H. Poincaré A (N. S.), 37:1 (1982), $1-28$.

[5] С. Н. Лакаев, Функи. анализ и его прил., 27:3 (1993), 15-28.

[6] J. Rauch, J. Funct. Anal., 35:3 (1980), 304-315.

[7] H. Tamura, Nagoya Math. J., 130 (1993), 55-83.

[8] Д. Р. Яфаев, Матем. сб., 94(136):4(8) (1974), 567-593.

[9] S. Albeverio, R. Høegh-Krohn, T. T. Wu, Phys. Lett. A, 83:3 (1971), 105-109.

[10] Yu. N. Ovchinnikov, I. M. Sigal, Ann. Physics, 123:2 (1989), 274-295.

[11] A. V. Sobolev, Commun. Math. Phys., 156:1 (1993), 101-126.

[12] S. Albeverio, S. N. Lakaev, Z. I. Muminov, Ann. Henri Poincaré, 5:4 (2004), 743-772.

[13] K. Winkler, G. Thalhammer, F. Lang, R. Grimm, J. Hesker Denschlag, K. J. Daley, A. Kantian, H. P. Büchler, P. Zoller, Nature, 441:7095 (2006), 853-856, arXiv: cond-mat/0605196.

[14] P. A. F. da Veiga, L. Ioriatti, M. O'Carroll, Phys. Rev. E, 66:1 (2002), 016130, 9 pp.

[15] V. Bach, P. W. De Siqueira, S. N. Lakaev, Bounds on the discrete spectrum of lattice Schrödinger operators, Preprint mp-arc 10-143, 2010, 45 pp.

Поступила в редакцию 1.03.2011 